\title{
Transcatheter Aortic Valve Replacement in Bicuspid Aortic Valve Disease: An Insight
}

\author{
Varshil Mehta ${ }^{1+}$, Nikhil Nalluri ${ }^{2+}$, Varun Kumar ${ }^{3}$, Nileshkumar Patel ${ }^{4}$, \\ Varunsiri Atti ${ }^{5}$, Arvin Narula ${ }^{4}$, Mauricio Cohen ${ }^{4}$
}

\author{
${ }^{1}$ Icahn School of Medicine at Mount Sinai, New York, ${ }^{2}$ Staten Island University Hospital, New York, \\ ${ }^{3} \mathrm{Mt}$. Sinai St. Luke's Roosevelt Hospital Center, New York, ${ }^{4}$ University of Miami/Miller School of Medicine, \\ Miami, Florida, ${ }^{5}$ Michigan State University, Michigan, USA \\ †Authors share equal contribution to this manuscript. \\ Address for correspondence: Varshil Mehta, Icahn School of Medicine at Mount Sinai, New York, USA. \\ E-mail: varshilmehta@gmail.com
}

\section{Abstract}

As per the current scenario, the role of transcatheter aortic valve implantation (TAVI) is controversial in bicuspid aortic valve (BAV) stenosis. All the randomized clinical trials comparing outcomes of TAVI with surgery, till date, have excluded patients with the BAV. Some of the observational studies have reported outcomes of TAVI in BAV stenosis patients who are not a surgical candidate. The recent advances in TAVI and its expansion into intermediate groups, which includes younger age groups sparks a debate on the efficacy and safety of TAVI in BAV. The purpose of the present article is to review the available literature regarding the feasibility, safety, and outcomes of TAVI in BAV stenosis.

\section{Keywords: Aortic stenosis, bicuspid aortic valve disease, transcatheter aortic valve implantation}

\section{Case Report}

Eighty-five year-old female patient with the medical history of diabetes, hyperlipidemia on statins, and chronic atrial fibrillation on Coumadin was referred to our institute for severe symptomatic aortic stenosis. She endorses that her condition has deteriorated over past year. She started noticing lower extremity swelling, two pillow orthopnea and fatigue on exertion. Echocardiogram at our institute showed severe aortic stenosis with a peak velocity of $4.1 \mathrm{~m} / \mathrm{sec}$, peak gradient 67, mean gradient $39 \mathrm{mmHg}$, and AVA $0.8 \mathrm{~cm}^{2}$. It was not clear that the patient has bicuspid or tricuspid aortic valve from the echocardiogram. After heart team discussion, it was decided that the patient is not a candidate for open surgical valve replacement because of her age and frailty. CT scan showed done as a part of TAVI work up showed: Bicuspid aortic valve.

\section{Introduction}

Bicuspid aortic valve (BAV)

BAV is defined as a spectrum of abnormal aortic valve morphology which consists of two functional cusps with $<3$ zones of parallel apposition between cusps. ${ }^{[1]}$ BAV is a common congenital disorder found in adults at a rate of $0.5-2 \%$ with a predilection towards males, ${ }^{[2-4]}$ with a demonstrated association with mutations in the NOTCH1 gene. ${ }^{[5]}$ Interestingly, its heritability is as high as $89 \%$, suggesting an autosomal dominant pattern of inheritance. ${ }^{[6-8]}$ Individuals with BAV are prone to develop aortic stenosis (AS) at an earlier age when compared to people with tricuspid aortic valve (TAV). Nonetheless, bicuspid valves also pose a risk of developing symptomatic AS in elderly patients. ${ }^{[9]}$ In fact, it is observed that its frequency is as high as 6\% of the population over 85 years of age. ${ }^{[10]}$

Approximately $20-50 \%$ of the patients with a BAV require aortic valve replacement in their lifetime. ${ }^{[11]}$ BAS is generally treated with surgical aortic valve replacement (SAVR) in patients $<70$ years of age. ${ }^{[9]}$ However, SAVR may not be a treatment option for patients with high surgical risk due to comorbidities or in the presence of absolute contraindications such as porcelain aorta (extensively calcified ascending aorta and/or aortic arch). Even though

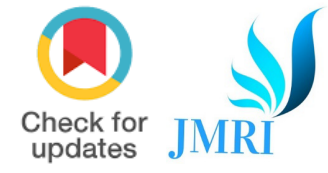

How to cite this article: Mehta $\mathrm{V}$, Nalluri N, Kumar V, Patel N, Atti V, Narula A, Cohen M. Transcatheter Aortic Valve Replacement in Bicuspid Aortic Valve Disease: An Insight. J Med Res Innov. 2019;3(2):e000180.

DOI:10.32892/jmri.180

Publication history: Received: 24-05-2019 Accepted: 10-07-2019

Published: 16-07-2019

Editor: Dr. Krutarth Shah

Copyright: Mehta $V$, Nalluri $\mathrm{N}$, Kumar V, Patel N, Atti V, Narula A, Cohen M. This is an open access article distributed under the terms of the Creative Commons Attribution License CCBY 4.0., which permits unrestricted use, distribution, and reproduction in any medium, provided the original author and source are credited.

Funding: NIL

Conflict of Interest: NIL

MK Medkrux 


\section{Mehta, et al.: Transcatheter aortic valve replacement in bicuspid aortic valve disease}

percutaneous balloon valvuloplasty can be an option in this population, AS reoccurs in the majority of the patients in the long-term. ${ }^{[12,13]}$

\section{Transcatheter aortic valve implantation (TAVI)}

TAVI is a minimally invasive procedure in which a new bioprosthetic (self-expandable or balloonexpandable) valve is inserted through catheter delivery system and implanted into aortic valve position without removing the old and diseased valve. ${ }^{[14]}$ Once the new valve is placed and expanded, it pushes the old valve leaflets from the site, and the tissue in the replacement valve takes over the job of regulating blood flow.

TAVI is performed through different approaches: Transfemoral, transapical, subclavian, direct aortic, and transcaval. ${ }^{[14]}$ Current ACC/AHA guidelines recommend TAVI as Class I for patients with prohibitive and high surgical risk(with a post-TAVR survival greater than 12 months), whereas Class II for patients with intermediate surgical risk.

The recent advances with the valve design, TAVI is being studied in lower-risk populations as well as to treat other disease states such as BAS, native aortic valve regurgitation, and failed bioprosthetic aortic valve stenosis. ${ }^{[15]}$

\section{BAV Anomaly, Associated Complications}

BAV anomaly has several variants. BAV without any unwanted tissue generally develops stenosis, whereas valves with unwanted tissue usually tend to develop valvular incompetence. ${ }^{[16]}$

AS is one of the most important complications of BAV and its incidence on autopsy ranges from $15 \%$ to $75 \%{ }^{[17]}$ Stenosis in BAV progresses with age, with fibrosis beginning in the second decade and calcification after the fourth decade. Among octogenarians and nonagenarians, who underwent SAVR, $22 \%$ and $18 \%$, respectively, were observed to have BAV. ${ }^{[9]}$

The most popular BAV classification was described by Sievers et al. in 2007. They identified three major types of BAV based on the number of raphae:

1. Type $0-$ No raphe

2. Type 1 - One raphe

3. Type 2-Two raphe.

They further subdivided these types into two subcategories based on:

1. Spatial arrangements of the cusp free edges (for example, BAV type 1 L/R means one raphe positioned between the left and right coronary sinuses) a. Type 0 - lateral and anterio-posterior

b. Type 1- L-R, R-N, and N-L

c. Type $2-L-R / R-N$.

( $\mathrm{L}=$ left coronary cusp, $\mathrm{R}=$ Right coronary cusp, and

$\mathrm{N}=$ Non coronary cusp)

2. Functional status - predominant insufficiency

(I), predominant stenosis (S), balanced

insufficiency and stenosis (B), and no

insufficiency and stenosis (No)

Most patients in the study had BAV Type 1 (88\%)

predominantly L-R, S category. ${ }^{[1]}$

Bicuspid aortopathy (i.e., dilation of any part or all segments of the proximal aorta from the aortic root to the aortic arch) is the most common nonvalvular finding found in approximately $50 \%$ of the affected patients. ${ }^{[3,18]}$ Abnormal dilation of the ascending aorta is generally found secondary to the abnormalities of the aortic media and becomes of significant surgical interest when the diameter of the aorta exceeds $4.5 \mathrm{~cm} .^{[18]}$ Aortic media changes are present independent of the valve functionality (normal, stenotic, or incompetent). The pathology of ascending aorta is considered to be an expression of the genetic basis of BAV and may signify the use of surgical intervention. ${ }^{[4]}$

\section{Current Management Scenario}

At present, echocardiography is the most important and common imaging modality used with regard to diagnosis and surveillance. Transthoracic echocardiography (TTE), when compared to surgical operative records or surgical pathology, demonstrated a specificity of $96 \%$ and sensitivity of $78 \%$ for the diagnosis. ${ }^{[19]}$ One study with multiplane transesophageal echocardiogram (TEE) and operative records observed a sensitivity of $87 \%$ and a specificity of $91 \% .{ }^{[20]}$ Furthermore, pre-operative echocardiography and intraoperative TEE are crucial not only for planning the surgical approach but also, assessing repair status, respectively, when valve repair is considered for BAV regurgitation. ${ }^{[21]}$

Because echocardiography is generally diagnostic, but it is limited by patient and operator related factors. Especially in the setting of heavy calcification, echocardiography can be challenging due to artifacts. Multidetector CT (MDCT) and magnetic resonance imaging (MRI) are emerging as alternatives for the diagnosis of the BAV.

Tanaka et al. reported a sensitivity of $94 \%$ and specificity of $100 \%$ for ECG gated MDCT in detecting BAV. ${ }^{[22]}$ Cardiac MRI could also play an important role. A recent article reported a specificity of 


\section{Mehta, et al.: Transcatheter aortic valve replacement in bicuspid aortic valve disease}

$95 \%$ and sensitivity of $100 \%$ with steady-state free-precession cine MRI in the correct classification of a valve as bicuspid. ${ }^{[23]}$

With regard to treatment options, there is a recent emergence of new data suggesting that medical therapy may help in slowing down the progression of expansion and dissection in patients with BAV and aortic aneurysm. ${ }^{[21]}$ Moreover, beta-blocker treatment now has been recommended as a Class Ila for BAV patients with an enlarged aorta. ${ }^{[24]}$ Angiotensin receptor blockade is also a Class Ila recommendation to reduce the dilatation of the aorta. ${ }^{[25]}$

However, SAVR currently remains the gold standard of treatment for patients with BAS $<70$ years of age. ${ }^{[9]}$ 2010 Guidelines for the Diagnosis and Management of Patients with Thoracic Aortic Disease suggested surgery as a Class I recommendation in patients with genetic syndromes which could possibly lead to aortic dissection and BAV, at aortic diameters from 4 to $5 \mathrm{~cm} .{ }^{[25]}$ However, surgical method has its own limitations where it cannot be done, for example, porcelain aorta (extensively calcified ascending aorta and/or aortic arch), hostile chest (patients with a history of sternotomy or radiation) or in patients who are suffering from comorbidities. ${ }^{[12,13]}$ Percutaneous aortic balloon valvotomy is of limited use due to high recurrence rates. ${ }^{[12]}$

The latest method, which is in growing in popularity for its ability to treat patients who are at high or prohibitive risk for surgery is TAVI.

\section{Should TAVI be used in BAV?}

Treatment of BAV disease with TAVI is yet considered as an off-label indication. Most randomized clinical trials have excluded patients with BAV stenosis. The specific concerns regarding TAVI for BAV include:

1. The asymmetry and heavy calcification involving the leaflets can prevent the valve from expanding properly, which might result in a para-valvular leak and high transvalvular gradients.

2. The elliptical annular anatomy of a BAV may lead to valve malpositioning. ${ }^{[26]}$

3. There is a risk of severe aortic regurgitation due to the disruption of the fused commissures.

4. The concomitant aortic disease increases the chances of rupture or dissection during the procedure.

5. Finally, if a valve is malpositioned or under expanded due to the above-mentioned challenges, it affects its long-term durability.
The bicuspid valves which are surgically excised typically demonstrate leaflet fusion (raphe) and extensive nodular calcification. The morphological distribution of calcific deposits in BAV leaflets is different as compared to that of tricuspid AS (TAS). ${ }^{[27]}$ As mentioned above, the extensive calcium deposition in the body of BAV leaflets and asymmetrical nature of the bicuspid aortic root could impair TAVI outcomes. ${ }^{[1,28]}$

In a retrospective study conducted by Mylotte et al., ${ }^{[29]}$ they have noticed that Guidewire crossing and transcatheter heart valve (THV) positioning were more difficult with bicuspid than TAV stenosis. However, the procedural results were acceptable and comparable to those reported in TAVI with TAS. ${ }^{[30-35]}$ The acute valve embolization occurred in $2.2 \%$ and conversion to SAVR occurred in $2.2 \%$, whereas the 30-day rates of VARC-defined device safety $(79.1 \%)$, efficacy (84.9\%), and success (89.9) were encouraging as well.

However, there are very few studies done in the past which support the use of TAVI in BAS. The first description of TAVI procedure in the BAV population was done and published by Wijesinghe et al., and it included 11 high-risk patients who were treated with a balloon-expandable prosthesis (EdwardsSAPIEN [Edwards Lifesciences, Irvine, California]). The mean age was $73.2 \pm 12.5$ and predicted the risk of mortality (PROM) was $4.4 \pm 2.6 \%$ according to the Society of Thoracic Surgeons score. TAVI was performed through the transfemoral (63.4\%) or transapical route $(36.4 \%)$ under general anesthesia. After the implantation, $18 \%$ of the patients had a moderate perivalvular leak (PVL), and the 30 days mortality was 18\%. At follow-up (median time: 208 days) mortality rate was found out to be $36.4 \%$, which was mainly driven by deaths occurring in the transapical group in which all four patients died. At follow up, all survivors at follow-up were either in Class I or Class II of New York Heart Association (NYHA). Limited by only one type of bioprosthesis, this study for the first time showed that TAVI in BAV is feasible in a selected group of patients. ${ }^{[36]}$

Yoon et al. in 2016 reported clinical outcomes of TAVI in BAS with regard to early and new-generation devices in the multicenter bicuspid TAVI registry. This registry enrolled 301 consecutive patients undergoing TAVI for BAS which included 199 patients $(71.1 \%)$ receiving early-generation devices (Sapien XT [Edwards Lifesciences, Irvine, California]: $n=87$; and CoreValve [Medtronic, Minneapolis, Minnesota]: ( $n=112$ ) and 102 patients (29.9\%) receiving new-generation devices (Sapien 3

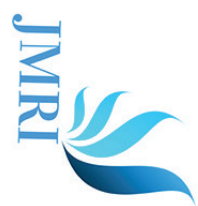




\section{Mehta, et al.: Transcatheter aortic valve replacement in bicuspid aortic valve disease}

[Edwards Lifesciences]: $n=91$; and Lotus [Boston Scientific, Marlboro ugh, Massachusetts: $(n=11)$. Mean age in the studied group was $77.0 \pm 9.2$ years, and the Society of Thoracic Surgeons score was $4.7 \pm 5.2$, which classified the population as intermediate surgical risk group. ${ }^{[37]}$ They also provided a comparison between the old and new generation devices, showing the superiority of new-generation over old-generation devices for the treatment of BAS (device success $92.2 \%$ vs. $80.9 \% ; P=0.01$, and second valve implantation $1 \%$ vs. $6.5 \% ; P=0.04$ ). All-cause mortality rates were $4.3 \%$ at 30 days and $14.4 \%$ at 1 year, which was comparable to that of TAVI for TAS in the general population. Moreover, there were no reported cases of moderate-to-severe paravalvular regurgitation with new-generation devices (Sapien 3 [Edwards Lifesciences] and Lotus [Boston Scientific, Marlborough, Massachusetts]) while the old generation devices had an incidence rate of $8.5 \%$ (Sapien XT [Edwards Lifesciences, Irvine, California]: $n=5$ (5.7\%); and CoreValve [Medtronic, Minneapolis, Minnesota]: $n=12(1))$. These results from Yoon et al. offered a reassurance that TAVI may indeed be a reasonable treatment option for such patients. ${ }^{[37]}$

Himbert et al. conducted a study to assess the results of TAVI in BAV with self-expandable valve Medtronic CoreValve system (MCS; Medtronic, Inc., Minneapolis, Minnesota) in which 15 patients with a mean age of $80 \pm 10$ and high mortality risk (Society of Thoracic Surgeons score $8 \pm 5$ ) were evaluated. The transfemoral approach was used in all except for one patient, and three procedures were performed under general anesthesia, which gave access for periprocedural trans-esophageal guidance. Although six patients required permanent pacemaker implantation (40\%), moderate PVL was seen only in one patient $(6.7 \%)$ and 30 days survival rate was $93 \%$. After a mean follow-up of $8 \pm 7$ months, two patients were dead (13\%), and the survivors (78.6\%) achieved an improvement in their functional status according to NYHA I or II. The results of this observation suggest that BAV can be also safely treated with a self-expandable prosthesis. However, a major disadvantage an absence of a control group (patients with tricuspid anatomy) and population size. ${ }^{[38]}$

The above issue was addressed in the next studies. Hayashida et al. compared the results of TAVI obtained in 21 BAV cases with the results achieved in 208 patients with a normal tricuspid valve. They only included patients who also underwent MDCT before TAVI along with other imaging modalities (TTE and TEE). However, no statistically significant differences were found between the groups in age, sex, and mortality risk. Similar results were observed in terms of 30 days mortality and moderate/severe PVL (4.8\% vs. $8.2 \% ; P=1.00$, and $19.0 \%$ vs. $14.9 \% ; P=0.54$, respectively). ${ }^{[39]}$

In a similar study by Costopoulos et al., comparing $\operatorname{BAV}(n=21)$ and TAV patients $(n=447)$ treated with Edwards or CoreValve bioprosthesis from November 2007 to December 2012, a difference in 30 days mortality and 1-year mortality $(14.2 \%$ vs. $3.6 \%$, $P=0.02$ ) and $10.5 \%$ vs. $7.4 \%, P=0.62$, respectively) was observed. The researchers concluded that even though TAVI in BAV patients with high surgical risk is feasible with acceptable mid-term outcomes, a careful selection of patients is necessary as patients with BAV showed lower early survival rates and device success. The reasons are mainly anatomic challenges posed by BAV patients. They have encountered one case of dissection, one case of valve migration, but the most common problem they encountered was a need for frequent postdilatation in BAV group ( $52.4 \%$ vs. $23.5 \%, P<0.01)$. This could be due to the asymmetric calcification seen BAV patients. This study is limited by its small sample size to draw any real-world long-term conclusions. ${ }^{[40]}$

Subsequently, in a sub-analysis of the German Aortic Valve Registry (GARY), a higher rate of PVL was reported amongst 38 BAV patients when compared with a large control group of 1357 TAV patients ( $25 \%$ vs. $15 \%$; $P=0.05$ ) which they attributed to the anatomic features of a BAV. ${ }^{[41]}$

In 2014-15, two relatively large, multicenter studies were carried out on TAVI in BAV. One of them was conducted by Mylotte et al., in which 139 BAV patients were included, and the procedural outcomes were evaluated for both self- and balloon-expandable bioprosthesis. The mean age of the patients enrolled was $78.0 \pm 8.9$ years, and the mean Society of Thoracic Surgeons (STS) PROM score was $4.9 \pm 3.4 \%$. A balloon-expandable THV (Sapien XT, Edwards Lifesciences, Inc., Irvine, California) and self-expandable THV (CoreValve, Medtronic, Inc., Minneapolis, Minnesota) were used in 48 patients (34.5\%) and 91 patients (65.5\%), respectively. Interestingly, the rate of moderate/ severe PVL in the studied group was $28.4 \%$ but was $17.4 \%$ in the subgroup in which valve size was based on MDCT. A trend toward a higher rate of relevant PVL in patients treated with a self-expandable valve (19.6 vs. 32.2; P1/40.11) was observed, but as per the 


\section{Mehta, et al.: Transcatheter aortic valve replacement in bicuspid aortic valve disease}

authors, it could be because of lower use of MDCT for valve sizing in this group. This study emphasizes on patient selection and pre-procedural imaging for better outcomes, especially in BAV. ${ }^{[29]}$

The other study was by Yousef et al., which looked into the outcomes of TAVI in 108 BAV patients. This study also confirmed the general feasibility and acceptable clinical outcomes of TAVI in BAV, in spite of the fact that a relatively high rate of reintervention was seen $(9.3 \%)$ because of either valve embolization or migration. ${ }^{[42]}$

The study conducted by Perlman et al., in 2016, it was concluded that TAVI in BAS using a newgeneration device (SAPIEN 3) was feasible and effective. Furthermore, valve performance was favorable, and no cases of moderate or severe AR were observed. This again was attributed to preprocedural CT annular sizing which was used to select an appropriately sized implant. The lack of significant PVL (>mild) could also be attributed to the improved sealing properties of the external sealing layer of the inflow portion of the SAPIEN 3 valve and improvements in the accuracy of valve positioning with an improved valve delivery system. In the study, 51 patients underwent TAVI using the SAPIEN 3 valve. The mean age in the patient group was $76.2 \pm 9.3$ years, and the predicted risk of mortality scores was $5.2 \pm 3.7 \%$ according to the Society of Thoracic Surgeons. The most common bicuspid valve type was type $1(82.3 \%)$ followed by type $0(11.8 \%)$ and type $2(1.9 \%)$. Post-dilation was performed in $7.8 \%$ of the cases. There were no cases of valve embolization or need for a second valve. The mean aortic gradient decreased from $49.4 \pm$ $16.0 \mathrm{~mm} \mathrm{Hg}$ to $11.2 \pm 4.7 \mathrm{~mm} \mathrm{Hg}$. Post-implantation AR was mild in $37 \%$ and none/trivial in $63 \%$. There were no cases of moderate or severe AR. At 30 days follow-up, 2 deaths (3.9\%), two major vascular complications were observed with 12 patients $(23.5 \%)$ requiring pacemaker implantation. ${ }^{[43]}$

The results of a recent international multicenter, observational study comparing outcomes of TAVI in bicuspid versus TAV were presented at the American college of cardiology conference. Here researchers compared outcomes of 561 patients with bicuspid AS and 4546 patients with TAS after propensity-score matching; assembling 546 pairs of patients with similar baseline characteristics. The study found that when compared to patients with TAS, patients with BAS had more frequent conversion to surgery $(2.0 \%$ vs. $0.2 \% ; P=0.006)$ and significantly lower device success rate $(85.3 \%$ vs. $91.4 \%$; $P=0.002$ ). Interestingly in these study early-generation devices (Sapien XT, CoreValve) were implanted in 320 patients with bicuspid and 321 patients with TAS, whereas new-generation devices (Sapien 3, Lotus, Evolut R) were implanted in 226 and 225 patients with bicuspid and TAS, respectively.

The early generation devices had a high incidence of moderate to severe PVL (15.4\%), whereas the incidence was low and comparable to TAS with newer generation devices (2.7\%). Among the early generation devices, the CoreValve had more moderate-to-severe PVL ( $19.4 \%$ vs. $10.5 \%, P=0.02)$. Among the BAS patients receiving early-generation devices, Sapien XT had more frequent aortic root injury (4.5\% vs. $0.0 \%, P=0.015)$. They concluded that TAVI in BAS was associated with similar prognosis but higher adverse procedural events compared to TAS. However, there were no differences with the new generation devices. The patients with newer-generation devices had acceptable and comparable procedural results to trisuspid TAVI and this was similar across different prostheses. The cumulative all-cause mortality rates at 2 years were comparable between bicuspid and TAS (17.2\% vs. $19.4 \%, P=0.28)$. The important finding of the study is that TAVI is feasible with certain BAS patients with newer generation valves (Sapien 3, Lotus, Evolut R). ${ }^{[4]}$

These studies are compared in Table 1. With the advancement of technology, it seems that TAVI can indeed be technically feasible in selected BAV patients, especially with the newer generation valves. However, a meticulous preprocedural assessment and planning are needed for better outcomes.

\section{Role of Pre-procedural Imaging in BAV TAVI}

In this new era of TAVI, there is a large role played by preprocedural assessment and imaging. The goals of preprocedural imaging are as follows:

1. Identify the patients with AS and confirm the severity.

2. Identifying patients suitable for TAVI.

3. Help in device selection (type and size) by defining the anatomic characteristics.

4. To develop a procedural plan (Vascular access/ fluoroscopic angulation).

The imaging methods vary among institution and individuals. ACC recently released expert consensus decision pathway for TAVI. The most commonly used imaging modalities are TTE, TEE, and CT scan.

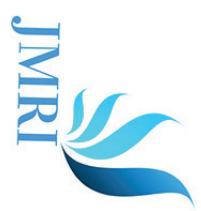


Mehta, et al.: Transcatheter aortic valve replacement in bicuspid aortic valve disease

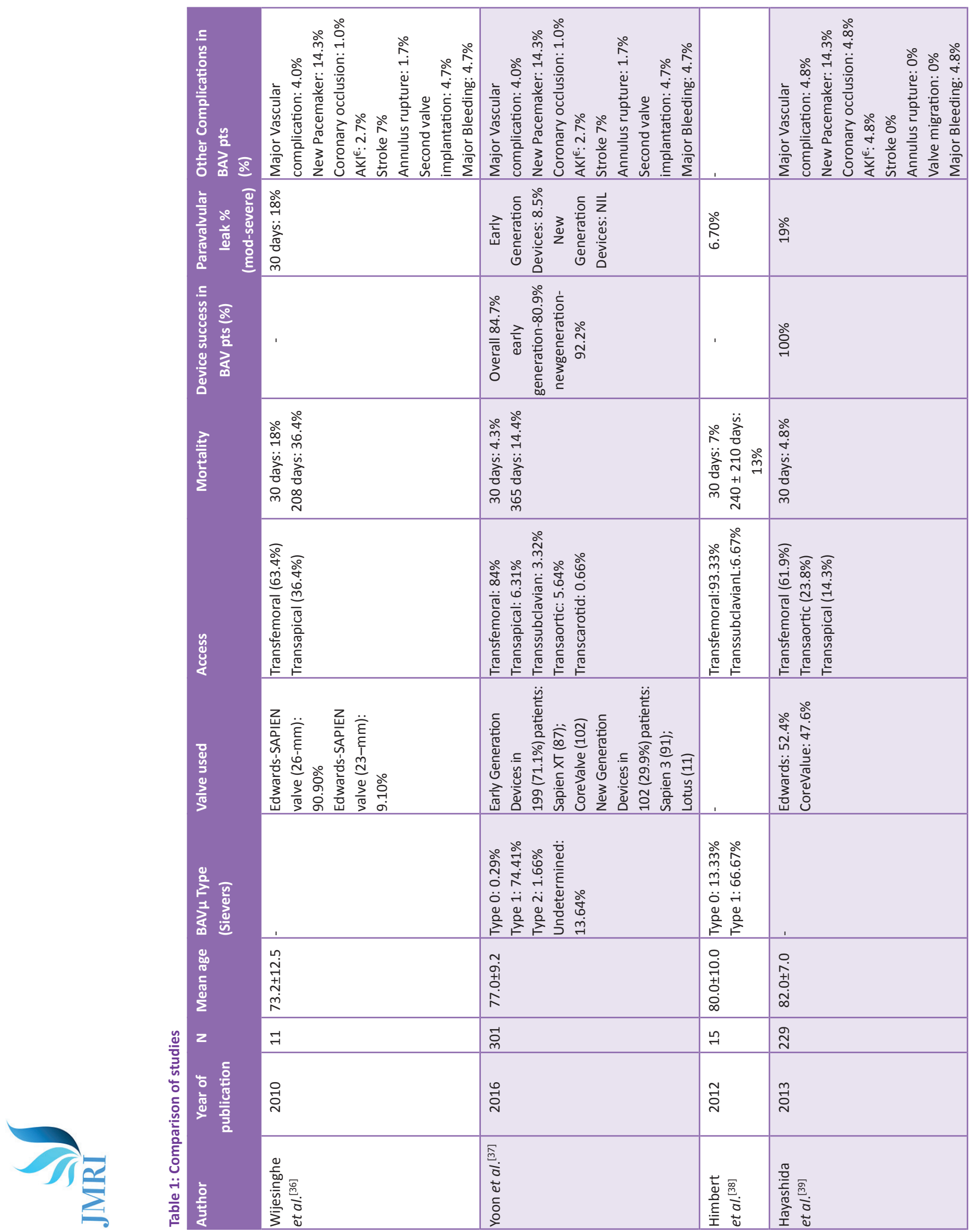


Mehta, et al.: Transcatheter aortic valve replacement in bicuspid aortic valve disease

\begin{tabular}{|c|c|c|c|c|}
\hline 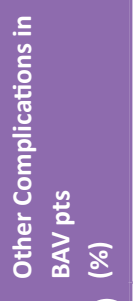 & 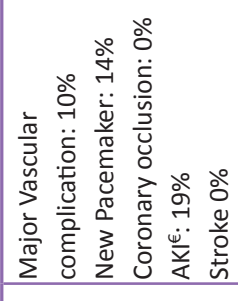 & 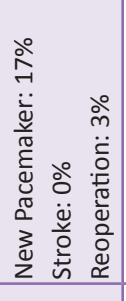 & 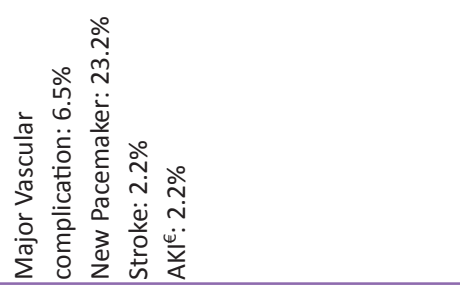 & 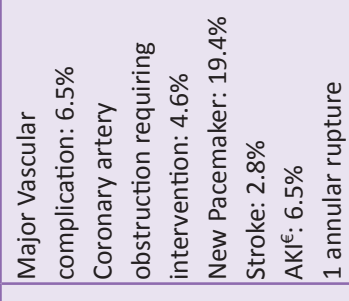 \\
\hline 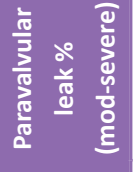 & & ڤั & 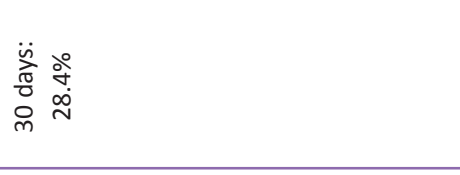 & 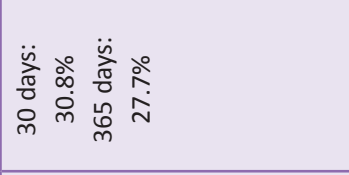 \\
\hline 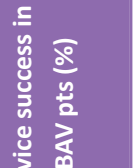 & ఏे & , & $\begin{array}{l}\text { தे } \\
\text { مे }\end{array}$ & 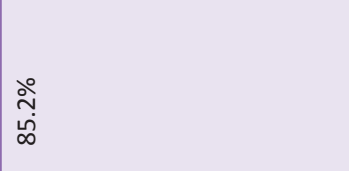 \\
\hline 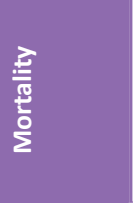 & 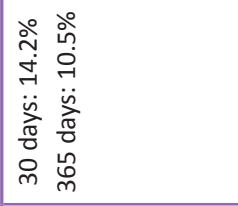 & 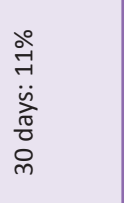 & 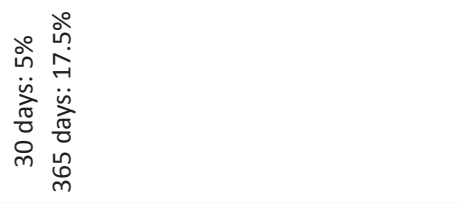 & 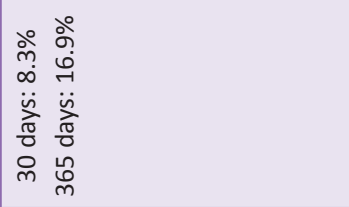 \\
\hline $\begin{array}{l}\tilde{y} \\
\mathbb{8} \\
\mathbb{8}\end{array}$ & & & 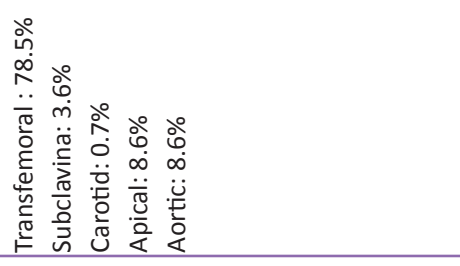 & 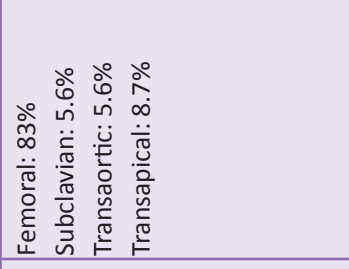 \\
\hline 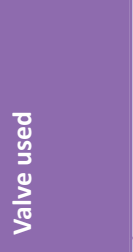 & & & 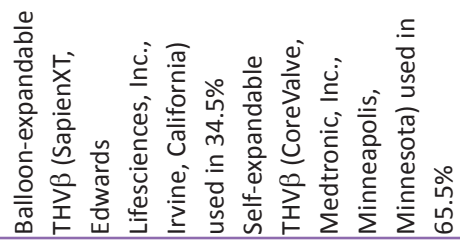 & 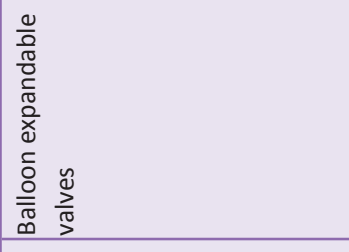 \\
\hline 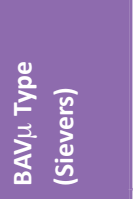 & & & 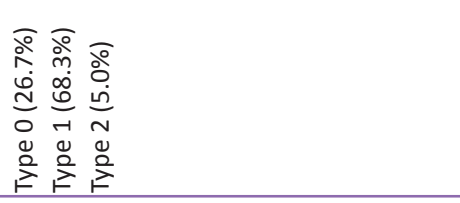 & 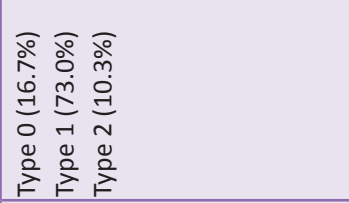 \\
\hline 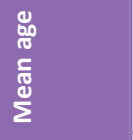 & $\begin{array}{l}\stackrel{1}{r} \\
+1 \\
\stackrel{1}{0} \\
0\end{array}$ & ' & $\begin{array}{l}0 \\
\infty \\
\infty \\
+1 \\
0 \\
\infty \\
\infty\end{array}$ & 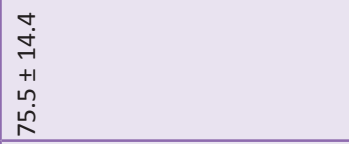 \\
\hline$z$ & $\vec{\lambda}$ & $\stackrel{\infty}{m}$ & $\underset{\nexists}{\vec{~}}$ & $\underset{-}{\infty}$ \\
\hline 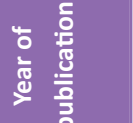 & 苛 & 吉 & 吉 & $\stackrel{n}{\stackrel{n}{\sim}}$ \\
\hline g & 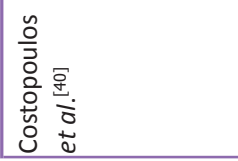 & 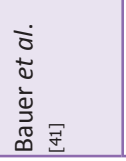 & 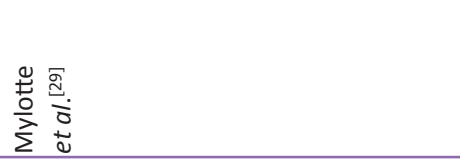 & 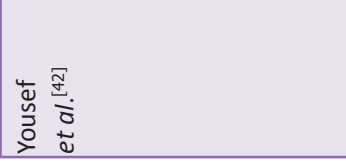 \\
\hline
\end{tabular}

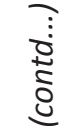


Mehta, et al.: Transcatheter aortic valve replacement in bicuspid aortic valve disease

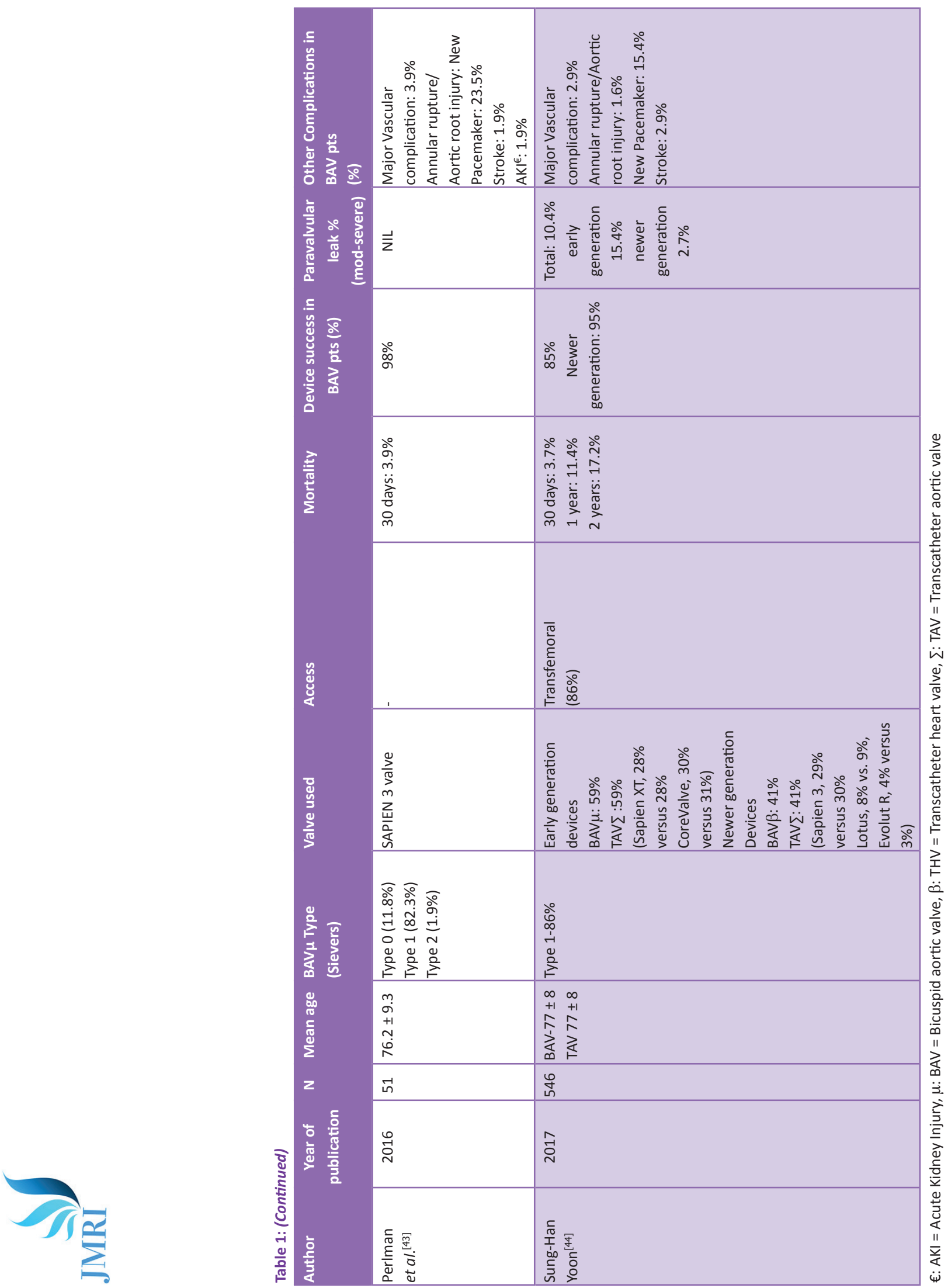




\section{Mehta, et al.: Transcatheter aortic valve replacement in bicuspid aortic valve disease}

TTE is routinely used for initial assessment of aortic valve type, AS severity, and left ventricular function. TTE or TEE imaging can sometimes be limited in detection of BAV by factors such as patient body habitus and excessive calcification. Although a 3D TEE can improve the detection of BAV, it is not always feasible in seriously ill patients. Hayasida et al. reported disparity regarding the diagnosis of BAV between MDCT and TTE/TEE. In their study, MDCT detected BAV in 21 patients (9.2\%), but TTE/TEE was able to pick only nine out the 21 cases. ${ }^{[39]}$

Given the favorable data coming out for TAVI in high-risk population, ${ }^{[45]}$ TAVI is being evaluated in the intermediate risk group. This risk group includes younger patients, whose frequency of BAV is higher than the elderly. ${ }^{[46]}$ With more BAV patients undergoing TAVI, there is a larger role for pre-procedural imaging to identify various anatomic as well as high-risk properties. This is achieved by a three-dimensional assessment of annular dimensions, which is commonly obtained by MDCT. ${ }^{[47,48]}$

MDCT helps in precise characterization of leaflet morphology, assessment of the symmetry of the aortic valve leaflets, and distribution of valvular calcification. All these mentioned factors are useful in selecting the type and size of the THV. MDCT also provides other anatomic information such as assessment of the left ventricular outflow tract, aortic root ("sinus of Valsalva"), determination of coronary heights, and identification of concomitant ascending aortopathy.

There are several classifications for BAV proposed earlier based on the pathological findings and echocardiography of which Sievers being the most popular one (e.g., Sievers et al. and Michelena et al.) [Table 2]. Most of the studies showed a high incidence of Sievers type 1 BAV. ${ }^{[1,44]}$ Each type of BAV has unique anatomic features which may affect the outcomes of TAVI. Mylotte et al. described higher rates of post-implantation aortic regurgitation in Sievers type 1 (34.2\%) compared to Sievers type 0 (13.3\%). ${ }^{[29]}$ In the advent of TAVI becoming more popular and widely used, there is a need for an alternative classification which can predict TAVI outcomes in BAV population. In the process, Jilaihawi et al. proposed a the TAVISpecific BAV classification using cardiac computed tomography analyzed by corelab (cedars-Sinai heart institute) to aid in pre-procedural planning in patients with BAV who are being evaluated for TAVI. ${ }^{[49]}$ The MDCT images in 91 of 130 patients were reviewed and a novel classification was proposed in an effort to predict procedural outcomes after TAVI. The classification is depicted in Figure 1.

Here, they came up with a simplified TAVI directed classification based on the leaflet morphologies and orientation.

The specified three morphologies of BAV

1. Tricommisural (23.3\%) - here one commissure is completely fused between two cusps, also known as functional or acquired type)

2. Bicommissural raphe type(55.6\%) - here two cusps are fused (usually a or proximal to the basal third of the sinus) by a fibrous or calcified ridge of various heights, does not reach the height of the commissure

3. Bicommissural non-raphe type $(21.1 \%)$ - the two cusps are completely fused from their basal origin by no visible seam here you see, only two commissures with no raphe or third commissure.

The leaflet orientation was divided based on the cusp fusion into:

1. Coronary cusp fusion - comparable to Sievers left-right for raphe type or anteroposterior for non-raphe type

2. Mixed noncoronary - coronary cusp fusion comparable with Sievers right-non or non- Left for raphe type or lateral for non-raphe type (1). They found no difference in overall rates of 30 day mortality $(3.8 \%)$, rates of moderate to severe AR $(19 \%$ vs. $19.5 \%$ vs. $15 \%)$, the rate for new pacemakers and cerebrovascular events (3.2\%) among anatomical subsets. The lack of outcomes correlation compared to Sievers classification (1), calls for an expansion of Jilaihawi et al.'s morphological classification. Assessment of the length of the raphe, the extent of calcification along the raphe could be important in predicting the outcomes of BAV TAVI.

One more important finding of Jilaihawi et al. was that the incidence of perivalvular aortic regurgitation (PAR) > moderate was $18.1 \%$ overall, but it was lower in patients who got a pre-procedural CT (11.5\%). Predictors of post-procedural aortic regurgitation included intercommissural distance for bicommissural BAV and lack of baseline CT sizing before the procedure. This further emphasized the need for pre-procedural imaging for better sizing and anatomic definition of the valve, which further helps in better outcomes. ${ }^{[49]}$

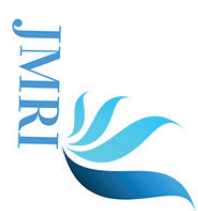


Mehta, et al.: Transcatheter aortic valve replacement in bicuspid aortic valve disease

Table 2: Different classifications of BAV

\begin{tabular}{|c|c|c|c|c|}
\hline Author (Ref. \#) & Specimens/Imaging test & $\begin{array}{l}\text { Number } \\
\text { of Cases }\end{array}$ & Criteria & Types \\
\hline \multirow[t]{2}{*}{ Roberts et al. ${ }^{[17]}$} & Surgical specimens & 59 & Cusp orientation & Anterior-posterior cusps \\
\hline & & & & Left-right cusps \\
\hline \multirow[t]{6}{*}{ Sabet et $a l_{.}^{[3]}$} & Pathology & 534 & Raphe orientation & RCC and LCC Fusion \\
\hline & & & & RCC and NCC fusion \\
\hline & & & & LCC and RCC fusion \\
\hline & & & $\begin{array}{l}\text { Relative cusp size/ } \\
\text { volume }\end{array}$ & Equal cusps \\
\hline & & & & Unequal cusps \\
\hline & & & & Thirds \\
\hline \multirow[t]{12}{*}{ Sievers et al. ${ }^{[1]}$} & Pathology & 304 & Number of raphae & Type 0 (none) \\
\hline & & & & Type 1 (one) \\
\hline & & & & Type 2 (two) \\
\hline & & & $\begin{array}{l}\text { Spatial position of } \\
\text { raphe/cusp }\end{array}$ & Anterior-posterior cusps \\
\hline & & & & Lateral cusps \\
\hline & & & & RCC and LCC fusion \\
\hline & & & & RCC and NCC fusion \\
\hline & & & & LCC and NCC fusion \\
\hline & & & $\begin{array}{l}\text { Functional status } \\
\text { of the valve }\end{array}$ & Stenosis \\
\hline & & & & Insufficiency \\
\hline & & & & Both \\
\hline & & & & None \\
\hline \multirow[t]{5}{*}{ Michelena et al. ${ }^{[11]}$} & Echocardiogram & - & $\begin{array}{l}\text { Location of the } \\
\text { commissures }\end{array}$ & Type 1: LCC and RCC fusion \\
\hline & & & & Type 2: RCC and NCC fusion \\
\hline & & & & Type 3: LCC and NCC fusion \\
\hline & & & Cusp symmetry & Symmetric \\
\hline & & & & Nonsymmetrical \\
\hline \multirow[t]{5}{*}{ Jilaihawi et al. ${ }^{[49]}$} & MDCT & 91 & $\begin{array}{l}\text { Leaflet } \\
\text { morphology }\end{array}$ & Tricommissural \\
\hline & & & & Bicommissural non-raphe type \\
\hline & & & & Bicommissural raphe type \\
\hline & & & Leaflet orientation & Coronary cusp fusion \\
\hline & & & & $\begin{array}{l}\text { Coronary/noncoronary cusp } \\
\text { fusion }\end{array}$ \\
\hline
\end{tabular}

\section{Back to the Case}

Based on CT scan finding, we decided to use $26 \mathrm{~mm}$ Evolut $\mathrm{R}$ valve for our patient. We accessed the common femoral artery with the help of fluoroscopy and ultrasound guidance. $26 \mathrm{~mm}$
Evolut $R$ valve was advanced into the $L V$. We noticed complete heart block, so we decided to pace at $80 \mathrm{bpm}$. We attempted two deployments, but the valve would dive in at the level of the left coronary into the LVOT. We decided to attempt 


\section{Mehta, et al.: Transcatheter aortic valve replacement in bicuspid aortic valve disease}

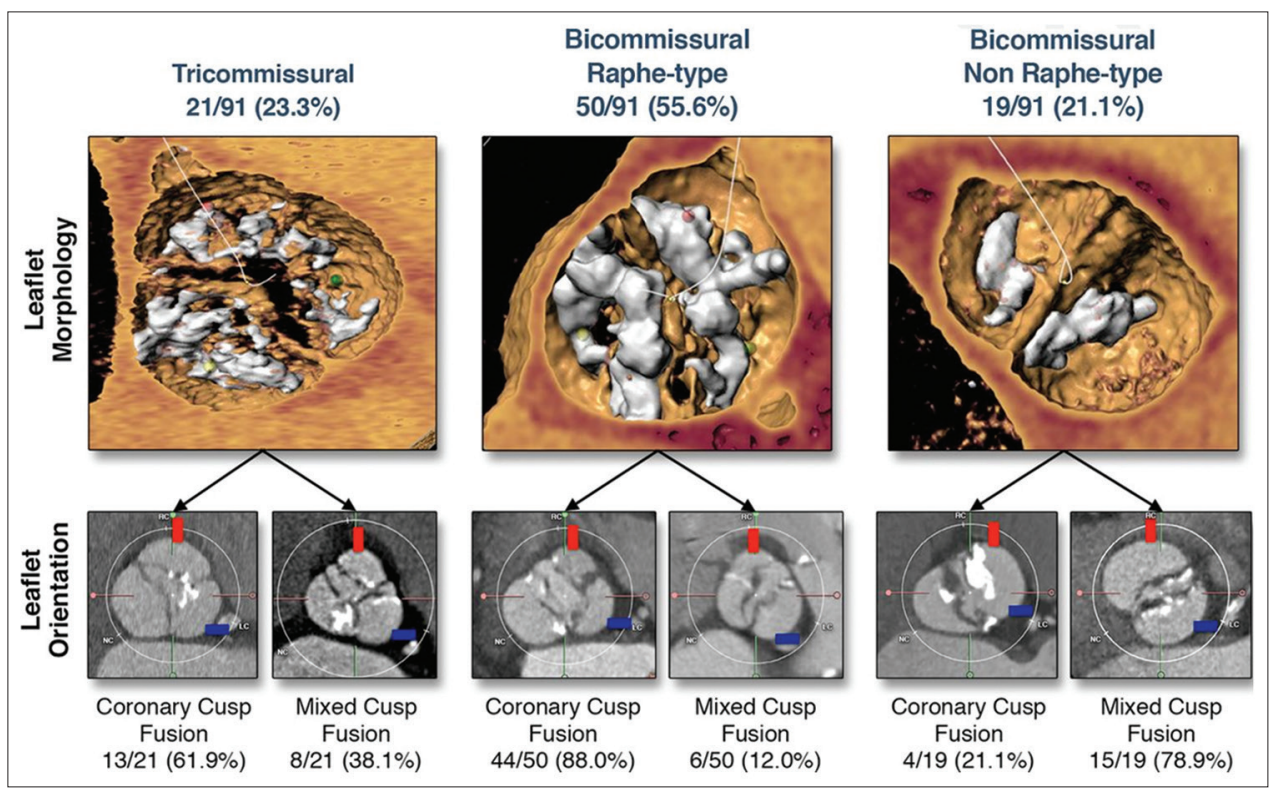

Figure 1: Transcatheter aortic valve replacement-specific bicuspid aortic valve classification

a third time with similar results. Even though we were not completely satisfied with the position of the valve; we had to deploy the valve as the patient became hemodynamically unstable. TEE revealed moderate PVL. The valve was post dilated the valve with a $20 \mathrm{~mm}$ true balloon. The BAV improved the expansion of the valve; however, there was still significant PVL. We decided to obtain right ulnar access (the radial artery had a loop and was occluded from a previous cath). We advanced a JR4 catheter an using a $10 \mathrm{~mm}$ Cook GooseNeck snare; we were able to snare one of the plates of the valve and slowly pulled back to a position where we achieved trivial PVL. We then noticed contrast stagnation and a contrast stain in the non-coronary cusp. We suspected a localized dissection of the non-coronary cusp. The patient became hypotensive and repeat CINE showed double contrast of the cardiac border. We confirmed this finding with TEE and performed pericardiocentesis with the removal of approximately $400 \mathrm{cc}$ of blood. The patient also received permanent pacemaker for complete heart block.

The patient was transferred to $\mathrm{CCU}$ in a hemodynamically stable condition. The patient became anuric and developed acute tubular necrosis from hypotension during the procedure. She initially required CVVHD for acute renal failure. Hospital course was further complicated by pneumonia and septic shock. The patient briefly required vasopressors for sepsis, but her pneumonia and sepsis improved with broad-spectrum antibiotics. Her renal function recovered completely and she no longer required CVVHD. She was transferred to the floor after 4 days in CCU. The patient improved dramatically and eventually discharged to inpatient rehab at a facility near her house. With subsequent follow-up, she is doing well and her symptoms of AS improved significantly.

\section{Conclusion}

With the available observational data, it seems that TAVI is indeed technically feasible in selected BAV patients with acceptable results, especially with new-generation devices. Most of the current data studies highlight the importance of preprocedural imaging (esp. MDCT) determining the outcome of TAVI in BAV patients. To further evaluate the role of TAVI in the BAV patients, we would need to follow the standards set by the PARTNER trial, which suggested the role of TAVI in the treatment of high risk and inoperable patients with TAS. More research into the subject involving more clinical trials should be done to compare the success rate and efficiency between TAVI and SAVR.

\section{References}

1. Sievers HH, Schmidtke C. A classification system for the bicuspid aortic valve from 304 surgical specimens. J Thorac Cardiovasc Surg 2007;133:1226-33.

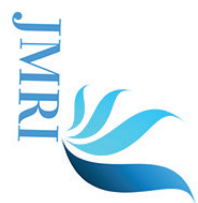




\section{Mehta, et al.: Transcatheter aortic valve replacement in bicuspid aortic valve disease}

2. Steinberger J, Moller JH, Berry JM, Sinaiko AR. Echocardiographic diagnosis of heart disease in apparently healthy adolescents. Pediatrics 2000;105:815-8.

3. Sabet HY, Edwards WD, Tazelaar HD, Daly RC. Congenitally bicuspid aortic valves: A surgical pathology study of 542 cases (1991 through 1996) and a literature review of 2,715 additional cases. Mayo Clin Proc 1999;74:14-26.

4. Siu SC, Silversides CK. Bicuspid aortic valve disease. J Am Coll Cardiol 2010;55:2789-800.

5. Garg V, Muth AN, Ransom JF, Schluterman MK, Barnes R, King IN, et al. Mutations in NOTCH1 cause aortic valve disease. Nature 2005;437:270-4.

6. Cripe L, Andelfinger G, Martin LJ, Shooner K, Benson DW. Bicuspid aortic valve is heritable. J Am Coll Cardiol 2004;44:138-43.

7. Huntington K, Hunter AG, Chan KL. A prospective study to assess the frequency of familial clustering of congenital bicuspid aortic valve. J Am Coll Cardiol 1997;30:1809-12.

8. Clementi M, Notari L, Borghi A, Tenconi R. Familial congenital bicuspid aortic valve: $A$ disorder of uncertain inheritance. Am J Med Genet 1996;62:336-8.

9. Roberts WC, Janning KG, Ko JM, Filardo G, Matter GJ. Frequency of congenitally bicuspid aortic valves in patients $\geq 80$ years of age undergoing aortic valve replacement for aortic stenosis (with or without aortic regurgitation) and implications for transcatheter aortic valve implantation. Am J Cardiol 2012;109:1632-6.

10. Lindroos M, Kupari M, Heikkilä J, Tilvis R. Prevalence of aortic valve abnormalities in the elderly: An echocardiographic study of a random population sample. J Am Coll Cardiol 1993;21:1220-5.

11. Michelena HI, Prakash SK, Corte AD, Bissell MM, Anavekar N, Mathieu P, et al. Bicuspid aortic valve: Identifying knowledge gaps and rising to the challenge from the international bicuspid aortic valve consortium (BAVCon). Circulation 2014;129:2691-704.

12. Transcatheter Aortic Valve Replacement: Indications and Outcomes. Available from: http://www. uptodate.com/contents/transcatheter-aorticvalve-replacement-indications-and-outcomes. [Last accessed on 2018 Oct 20].

13. Iung B, Cachier A, Baron G, Messika-Zeitoun D, Delahaye $\mathrm{F}$, Tornos $\mathrm{P}$, et al. Decision-making in elderly patients with severe aortic stenosis: Why are so many denied surgery? Eur Heart J 2005;26:2714-20.

14. American Heart Association. What is TAVI?. Available from: http://www.heart.org/HEARTORG/ Conditions/More/HeartValveProblemsandDisease/ What-is-TAVI_UCM_450827_Article.jsp\#. WAviXeArLIU. [Last accessed on 2016 Oct 22].

15. Praz F, Windecker S, Huber C, Carrel T,
Wenaweser P. Expanding indications of transcatheter heart valve interventions. JACC Cardiovasc Interv 2015;8:1777-96.

16. Roberts WC, Ko JM. Frequency by decades of unicuspid, bicuspid, and tricuspid aortic valves in adults having isolated aortic valve replacement for aortic stenosis, with or without associated aortic regurgitation. Circulation 2005;111:920-5.

17. Roberts WC. The congenitally bicuspid aortic valve. A study of 85 autopsy cases. Am J Cardiol 1970;26:72-83.

18. Verma S, Siu SC. Aortic dilatation in patients with bicuspid aortic valve. N Engl J Med 2014;370:1920-9.

19. Brandenburg RO Jr., Tajik AJ, Edwards WD, Reeder GS, Shub C, Seward JB, et al. Accuracy of 2-dimensional echocardiographic diagnosis of congenitally bicuspid aortic valve: Echocardiographic-anatomic correlation in 115 patients. Am J Cardiol 1983;51:1469-73.

20. Espinal $M$, Fuisz AR, Nanda NC, Aaluri SR, Mukhtar O, Sekar PC, et al. Sensitivity and specificity of transesophageal echocardiography for determination of aortic valve morphology. Am Heart J 2000;139:1071-6.

21. Kiefer TL, Wang A, Hughes GC, Bashore TM. Management of patients with bicuspid aortic valve disease. Curr Treat Options Cardiovasc Med 2011;13:489-505.

22. Tanaka R, Yoshioka K, Niinuma H, Ohsawa S, Okabayashi $\mathrm{H}$, Ehara $\mathrm{S}$, et al. Diagnostic value of cardiac CT in the evaluation of bicuspid aortic stenosis: Comparison with echocardiography and operative findings. AJR Am J Roentgenol 2010;195:895-9.

23. Gleeson TG, Mwangi I, Horgan SJ, Cradock A, Fitzpatrick P, Murray JG, et al. Steady-state freeprecession (SSFP) cine MRI in distinguishing normal and bicuspid aortic valves. J Magn Reson Imaging 2008;28:873-8.

24. Warnes CA, Williams RG, Bashore TM, Child JS, Connolly HM, Dearani JA, et al. ACC/AHA 2008 guidelines for the management of adults with congenital heart disease: A report of the american college of cardiology/American heart association task force on practice guidelines (Writing committee to develop guidelines on the management of adults with congenital heart disease). Developed in collaboration with the american society of echocardiography, heart rhythm society, international society for adult congenital heart disease, society for cardiovascular angiography and interventions, and society of thoracic surgeons. J Am Coll Cardiol 2008;52:e143-e263.

25. Hiratzka LF, Bakris GL, Beckman JA, Bersin RM, Carr VF, Casey DE Jr., et al 2010 ACCF/AHA/AATS/ ACR/ASA/SCA/SCAI/SIR/STS/SVM guidelines for the diagnosis and management of patients with thoracic aortic disease: A report of the american 


\section{Mehta, et al.: Transcatheter aortic valve replacement in bicuspid aortic valve disease}

college of cardiology foundation/American heart association task force on practice guidelines, american association for thoracic surgery, american college of radiology, american stroke association, society of cardiovascular anesthesiologists, society for cardiovascular angiography and interventions, society of interventional radiology, society of thoracic surgeons, and society for vascular medicine. Circulation 2010;121:e266-369.

26. Zegdi R, Blanchard D, Azarine A, Folliguet T, Fabiani JN. Elliptical shape of a SAPIEN XT prosthesis deployed in a patient with bicuspid aortic valve stenosis. J Heart Valve Dis 2012;21:764-6.

27. Isner JM, Chokshi SK, DeFranco A, Braimen J, Slovenkai GA. Contrasting histoarchitecture of calcified leaflets from stenotic bicuspid versus stenotic tricuspid aortic valves. J Am Coll Cardiol 1990;15:1104-8.

28. Michelena HI, Khanna AD, Mahoney D, Margaryan E, Topilsky Y, Suri RM, et al. Incidence of aortic complications in patients with bicuspid aortic valves. JAMA 2011;306:1104-12.

29. Mylotte D, Lefevre T, Søndergaard L, Watanabe $Y$, Modine T, Dvir D, et al. Transcatheter aortic valve replacement in bicuspid aortic valve disease. J Am Coll Cardiol 2014;64:2330-9.

30. Leon $M B$, Smith $C R$, Mack $M$, Miller DC, Moses JW, Svensson LG, et al. Transcatheter aortic-valve implantation for aortic stenosis in patients who cannot undergo surgery. N Engl J Med 2010;363:1597-607.

31. Smith CR, Leon MB, Mack MJ, Miller DC, Moses JW, Svensson LG, et al. Transcatheter versus surgical aortic-valve replacement in high-risk patients. N Engl J Med 2011;364:2187-98.

32. Makkar RR, Jilaihawi $H$, Chakravarty $T$, Fontana GP, Kapadia S, Babaliaros V, et al. Determinants and outcomes of acute transcatheter valve-in-valve therapy or embolization: A study of multiple valve implants in the U.S. PARTNER trial (Placement of aoRTic traNscathetER valve trial edwards SAPIEN transcatheter heart valve). J Am Coll Cardiol 2013;62:418-30.

33. Gilard $M$, Eltchaninoff $H$, lung $B$, Donzeau-Gouge $P$, Chevreul K, Fajadet J, et al. Registry of transcatheter aortic-valve implantation in high-risk patients. N Engl J Med 2012;366:1705-15.

34. Roy DA, Schaefer U, Guetta V, Hildick-Smith D, Möllmann $\mathrm{H}$, Dumonteil $\mathrm{N}$, et al. Transcatheter aortic valve implantation for pure severe native aortic valve regurgitation. J Am Coll Cardiol 2013;61:1577-84.

35. Ussia GP, Barbanti M, Petronio AS, Tarantini G, Ettori F, Colombo A, et al. Transcatheter aortic valve implantation: 3-year outcomes of selfexpanding coreValve prosthesis. Eur Heart J 2012;33:969-76.
36. Wijesinghe N, Ye J, Rodés-Cabau J, Cheung A, Velianou JL, Natarajan MK, et al. Transcatheter aortic valve implantation in patients with bicuspid aortic valve stenosis. JACC Cardiovasc Interv 2010;3:1122-5.

37. Yoon SH, Lefèvre $\mathrm{T}$, Ahn JM, Perlman GY, Dvir D, Latib A, et al. Transcatheter aortic valve replacement with early-and new-generation devices in bicuspid aortic valve stenosis. J Am Coll Cardiol 2016;68:1195-205.

38. Himbert D, Pontnau F, Messika-Zeitoun D, Descoutures F, Détaint D, Cueff C, et al. Feasibility and outcomes of transcatheter aortic valve implantation in high-risk patients with stenotic bicuspid aortic valves. Am J Cardiol 2012;110:877-83.

39. Hayashida K, Bouvier E, Lefèvre T, Chevalier B, Hovasse $\mathrm{T}$, Romano $\mathrm{M}$, et al. Transcatheter aortic valve implantation for patients with severe bicuspid aortic valve stenosis. Circ Cardiovasc Interv 2013;6:284-91.

40. Costopoulos C, Latib A, Maisano F, Testa L, Bedogni F, BuchananL,etal.Comparisonofresultsoftranscatheter aortic valve implantation in patients with severely stenotic bicuspid versus tricuspid or nonbicuspid valves. Am J Cardiol 2014;113:1390-3.

41. Bauer T, Linke A, Sievert $H$, Kahlert $P$, Hambrecht $R$, Nickenig $G$, et al. Comparison of the effectiveness of transcatheter aortic valve implantation in patients with stenotic bicuspid versus tricuspid aortic valves (from the german TAVI registry). Am J Cardiol 2014;113:518-21.

42. Yousef A, Simard T, Webb J, Rodés-Cabau J, Costopoulos C, Kochman J, et al. Transcatheter aortic valve implantation in patients with bicuspid aortic valve: A patient level multi-center analysis. Int J Cardiol 2015;189:282-8.

43. Perlman GY, Blanke P, Dvir D, Pache G, Modine T, Barbanti $\mathrm{M}$, et al. Bicuspid aortic valve stenosis: Favorable early outcomes with a next-generation transcatheter heart valve in a multicenter study. JACC Cardiovasc Interv 2016;9:817-24.

44. Yoon SH, Bleiziffer S, De Backer O, Delgado V, Arai T, Ziegelmueller J, et al. Procedural and clinical outcomes in transcatheter aortic valve replacement for bicuspid versus tricuspid aortic valve stenosis. J Am Coll Cardiol 2017;69:2579-89.

45. Adams DH, Popma JJ, Reardon MJ, Yakubov SJ, Coselli JS, Deeb GM, et al. Transcatheter aortic-valve replacement with a self-expanding prosthesis. $\mathrm{N}$ Engl J Med 2014;370:1790-8.

46. Webb J, Gerosa G, Lefèvre T, Leipsic J, Spence M, Thomas $\mathrm{M}$, et al. Multicenter evaluation of a nextgeneration balloon-expandable transcatheter aortic valve. J Am Coll Cardiol 2014;64:2235-43.

47. Popma JJ, Adams DH, Reardon MJ, Yakubov SJ, Kleiman NS, Heimansohn D, et al. Transcatheter aortic valve replacement using a self-expanding

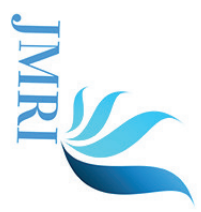


Mehta, et al.: Transcatheter aortic valve replacement in bicuspid aortic valve disease

bioprosthesis in patients with severe aortic stenosis at extreme risk for surgery. J Am Coll Cardiol 2014;63:1972-81.

48. Reardon MJ, Adams DH, Kleiman NS, Yakubov SJ, Coselli JS, Deeb GM, et al. 2-year outcomes in patients undergoing surgical or self-expanding transcatheter aortic valve replacement. J Am Coll Cardiol 2015;66:113-21.

49. Jilaihawi $H$, Chen M, Webb J, Himbert D, Ruiz CE, Rodés-Cabau J, et al. A bicuspid aortic valve imaging classification for the TAVR era. JACC Cardiovasc Imaging 2016;9:1145-58. 